\title{
Magnetic PDF analysis: A tool for making geometrically frustrated magnets less frustrating
}

\author{
Benjamin Frandsen $^{\mathrm{a}}$

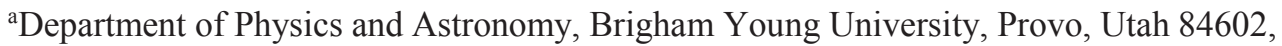 \\ benfrandsen@byu.edu
}

In geometrically frustrated magnets, the arrangement of magnetic moments within the crystal lattice prevents the simultaneous satisfaction of competing magnetic interactions. This hinders magnetic ordering and may lead to a variety of exotic phenomena, such as the formation of macroscopically degenerate spinliquid ground states lacking any long-range magnetic order. Even if long-range order does eventually form, frustrated magnets often display unusual sensitivity to perturbations, strong magnetic fluctuations above the ordering temperature, and other interesting behaviors. Experimental studies of the short-range magnetic correlations in frustrated magnets have historically been challenging - even frustrating, one might saysince conventional methods are typically only effective for determining long-range magnetic order. Here, I will provide two examples showing how magnetic pair distribution function (mPDF) analysis [1,2], a relatively new method of analyzing neutron scattering data to probe short-range magnetic correlations in real space, is a promising method for the quantitative investigation of geometrically frustrated systems. First, I will discuss our mPDF analysis of a new family of pyrochlore-type magnets based on transition metals and fluorine, where we recover the real-space pattern of short-range-ordered magnetic ground state from powder diffraction data [3]. Second, I will showcase the utility of combined atomic and magnetic pair distribution function analysis by unraveling the nature of the unusual magnetic transition in the triangular lattice antiferromagnet $\mathrm{NaMnO}_{2}$. Together, these examples teach us that geometrically frustrated magnets need not be so frustrating after all, as long as we use the right tools.

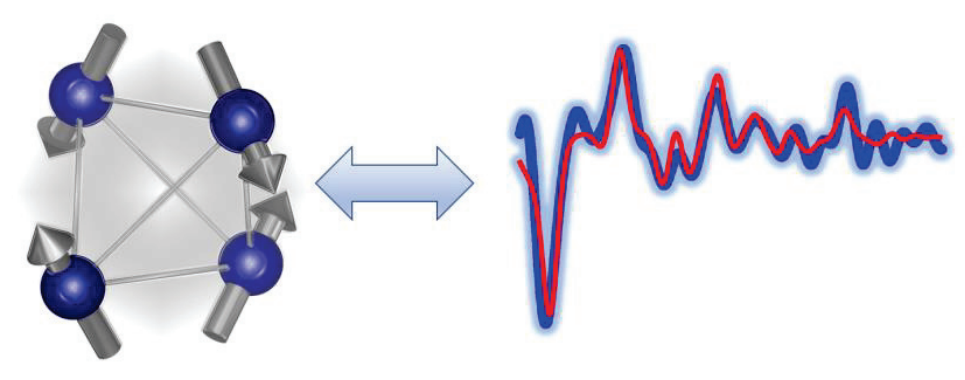

Fig. 1. Determining local magnetic correlations from magnetic pair distribution function analysis.

$\underline{\text { References }}$

[1] Frandsen, Yang, and Billinge, Acta Crystallographica A70, 3 (2014).

[2] Frandsen and Billinge, Acta Crystallographica A71, 325 (2015).

[3] Frandsen et al, Phys. Rev. Materials 1, 074412 (2017). 